\title{
Verrucous Nevus
}

National Cancer Institute

\section{Source}

National Cancer Institute. Verrucous Nevus. NCI Thesaurus. Code C4674.

A benign wart-like, pigmented skin lesion appearing on various parts of the body at birth or early in childhood, usually in linear groupings. 